REGARDS

SUR LEECONOMIE ALLEMANDE

BULLETIN ECONOMIQUE DU CRAC

\section{Regards sur l'économie allemande}

Bulletin économique du CIRAC

$93 \mid 2009$

Varia

\title{
Rhénanie du Nord-Westphalie : émergence d'un cluster médias
}

Isabelle Bourgeois

\section{OpenEdition}

\section{Journals}

Édition électronique

URL : http://journals.openedition.org/rea/3887

DOI : 10.4000/rea.3887

ISBN : 978-2-8218-0882-9

ISSN : 1965-0787

Éditeur

CIRAC

Édition imprimée

Date de publication : 1 octobre 2009

Pagination : 5-15

ISSN : 1156-8992

Référence électronique

Isabelle Bourgeois, «Rhénanie du Nord-Westphalie : émergence d'un cluster médias », Regards sur l'économie allemande [En ligne], 93 | octobre 2009, mis en ligne le 01 octobre 2011, consulté le 30 avril 2019. URL : http://journals.openedition.org/rea/3887 ; DOI : 10.4000/rea.3887 


\title{
Rhénanie du Nord-Westphalie : émergence d'un cluster médias
}

\author{
Isabelle Bourgeois
}

Le $1^{\text {er }}$ octobre 2009, le gouvernement de la Rhénanie du Nord-Westphalie (NRW) a lancé son deuxième appel à projets dans le cadre du programme Medien.NRW dédié à l'innovation dans le secteur des médias - plus précisément, à l'institutionnalisation d'un nouveau pôle de compétitivité alliant technologies de l'information et audiovisuel, supports et contenus : le Mediencluster NRW. Considérant que, du fait du processus de convergence, les médias sont une "branche clef » à forte dynamique de croissance et entrevoyant, de ce fait, un effet-levier indéniable pour le potentiel d'innovation du Land, la Rhénanie du Nord-Westphalie accorde aujourd'hui à l'excellence médiatique la même priorité qu'à celle en matière de biotechnologies, de nanotechnologies ou de logistique.

La constitution de ce cluster médias, officiellement annoncée en mars 2007, s'inscrit dans le cadre du programme de politique structurelle régionale que mène le Land. Au titre de la politique de cohésion de l'UE pour la période 2007-2013, il bénéficie de 1,28 milliard $€$ issus du Fonds EFRE (objectif 2). L'une des trois priorités de ce programme dénommé "NRW Ziel 2-Programm (EFRE) " est le développement de "l'innovation et de l'économie du savoir » qui se traduit notamment par la création de 16 pôles de compétitivité, tous dans les secteurs traditionnellement considérés comme à fort potentiel d'innovation (technologies médicales, environnementales, énergie, TIC...), sauf un, dédié aux médias (et un autre, dédié aux industries culturelles et créatives).

S'il peut surprendre dans un contexte de politique de reconversion industrielle et de promotion des technologies de pointe, le choix du secteur des médias est pourtant mûrement réfléchi. D'un côté, il décline à l'échelon d'un Land la nouvelle approche de la politique de cohésion territoriale communautaire placée sous le signe de l'innovation ("fléchage Lisbonne »). Et dans ce cadre, il met en œuvre le changement de paradigme de cette politique qui vise désormais à développer la compétitivité territoriale grâce à la valorisation des atouts existants. D'un autre côté, il prolonge les politiques des gouvernements précédents. L'idée d'un cluster médias n'est en effet pas née ex nihilo : en l'espace de deux décennies, NRW est devenu le premier site médiatique allemand. Dorénavant, il s'agit d'en optimiser les atouts en favorisant le rapprochement en réseau des divers acteurs concernés, donc en cultivant un terreau favorable à la créativité ; autrement dit : un climat propice à l'innovation. Par son approche, cette politique est elle-même innovante, puisqu'elle conjugue les moyens traditionnels de la politique structurelle régionale (dont le soutien aux PME) avec une approche élargie du concept d'innovation (incluant le capital humain ; voir REA 90/09), mais aussi avec les prémisses d'une réorientation de la politique structurelle communautaire. Car plus ou moins implicitement, le Mediencluster NRW mise sur la contribution du secteur culturel et créatif à la compétitivité de l'industrie et à l'attractivité des territoires.

\section{NRW : $1^{\text {er }}$ site médiatique allemand}

Le secteur des médias, puisqu'il appartient au domaine de la culture et que, au sein du partage des compétences législatives dans l'Allemagne fédérale, celui-ci relève de la souveraineté des Länder, est par essence polycentrique. La RFA ne connaît donc pas de centralisation des activités médiatiques dans la capitale. Et 
Objectif : rattraper et supplanter la Bavière en termes d'innovation

Mise en œuvre précoce de l'image valorisante des médias pour un Land en reconversion de même qu'on observe une concurrence nord-sud entre les 'poids lourds' industriels (NRW, Bade-Wurtemberg, Bavière et Hesse), deux grands sites médiatiques s'affrontent outre-Rhin: la Rhénanie du Nord-Westphalie et la Bavière qu'elle a détrônée en l'espace de vingt ans, en termes d'emplois (80 414 contre 68628 en Bavière) comme de production audiovisuelle, dont un tiers (en minutes) est issue de NRW. Ces deux 'poids lourds' médiatiques sont suivis par le Bade-Wurtemberg, Berlin/Brandebourg et Hambourg. Comme dans l'industrie, on y observe des spécialisations sectorielles, ainsi que le révèle par exemple la répartition des emplois soumis à cotisations sociales dans les activités du périmètre traditionnel des médias : radio, TV, livre, presse quotidienne et périodique, cinéma, musique et publicité. En NRW dominent la publicité (31\%), la presse quotidienne (21\%) et l'audiovisuel (17\% ; données 2007 ; me:mi, 2007). La structure des médias bavarois s'en distingue seulement par une part inférieure des activités publicitaires $(22 \%)$. Le secteur phare de la région de Berlin/Brandebourg est le cinéma (25\%), celui de Hambourg la presse périodique.

Dans la tradition du benchmarking allemand, où la Bavière et la Rhénanie du Nord-Westphalie s'affrontent pour tenir le leadership en matière de compétitivité économique et d'innovation, le développement de l'excellence médiatique devient aujourd'hui un axe stratégique. Alors que, avec près de $18 \%$ du PIB allemand, la Bavière n'est que la seconde économie de la RFA, elle dépasse de loin la NRW en matière d'innovation. Si on prend en considération le seul critère de la part du PIB que les Länder consacrent chacun à la R\&D, les Länder du sud sont de loin les plus performants : Bade-Wurtemberg $(4,31 \%)$ et Bavière $(2,92 \%)$, suivis par la Hesse $(2,64 \%$; données 2006, RWI, 2008). La NRW, où se concentre le cinquième (18 millions) de la population de l'Allemagne, et dont le PIB, équivalent à celui des Pays-Bas, pèse un bon cinquième (22\%) du PIB allemand, n'est plus aujourd'hui que le troisième site industriel de RFA. Et, avec 1,83 \% du PIB consacré à la $R \& D$, il ne figure pas parmi les économies les plus intensives en R\&D en comparaison des Länder du sud - ni non plus par rapport à la moyenne allemande $(2,54 \%)$. II est vrai que cette région anciennement de monoculture industrielle (charbon et acier) n'a pas encore achevé sa reconversion, bien que son économie se soit largement diversifiée au fil du temps et, surtout, tertiarisée : les services y représentent plus de $70 \%$ du PIB (un peu moins en moyenne allemande). Or ces services sont largement liés à l'industrie. Le Land ne s'est donc pas désindustrialisé ; au contraire, les mutations des activités ont donné naissance à une industrie de services. Le secteur de la logistique, où la NRW est leader incontesté, mais aussi l'essor des centres d'appel, en sont des exemples patents. Mais ces activités ne sont pas les plus intensives en R\&D.

Cette tertiarisation est le résultat d'une politique structurelle de longue haleine (Bourgeois, 2007), délibérément tournée vers le tertiaire - et savamment médiatisée. Dès le milieu des années 1980, le gouvernement du Land (alors SPD), mettant à profit à la fois l'effervescence technologique et industrielle qui caractérise le secteur des télécommunications depuis les années 1970 (développement de nouveaux vecteurs, supports, standards et services) et la libéralisation de l'audiovisuel en cours dans l'UE depuis le début des années 1980 (contenus), place en effet délibérément sa politique de reconversion sous le signe du... show biz. Conforté notamment par la présence à Gütersloh du puissant groupe Bertelsmann en pleine diversification, et par le lancement à Dortmund en 1984 d'un des 4 réseaux câblés pilotes allemands où s'expérimentaient les 'nouveaux médias' de l'époque, il mise alors sur l'image attractive (contenus) et modernisatrice (nouvelles technologies) des médias pour valoriser sa stratégie de 'révolution' structurelle vers une économie high-tech. Le concept de 'société de l'information' n'avait pas encore été forgé, mais les prémisses s'en dessinaient déjà. Et dans l'effort collectif de restructuration, la concurrence entre les villes - trois surtout - pour la promotion de leur site a joué un rôle décisif: Düsseldorf, capitale du Land et siège de l'opérateur de téléphonie mobile Vodafone (issu en 2000 d'une OPA sur le sidérurgiste Mannesmann qui se diversifiait dans ce secteur), Bonn, capitale de 
la RFA d'avant l'Unité et siège de Deutsche Telekom issu de l'ex-ministère fédéral des P\&T, et enfin Cologne, où se concentraient radio et TV publiques.

C'est Cologne qui a entrevu la première le potentiel de dynamisation régionale inhérent aux médias - en termes d'emplois, mais aussi d'image. Elle a su attirer sur son site le siège de la nouvelle chaîne privée RTL, créée conjointement par la société luxembourgeoise CLT et le groupe Bertelsmann (en partie fusionnés depuis dans RTL Group). Petit à petit, la production s'est installée autour de la diffusion, faisant de Hürth près de Cologne un des principaux centres de production audiovisuelle d'Allemagne, au plus près d'un riche gisement de créativité. L'effet boule de neige était lancé, Cologne est devenue la capitale médiatique du Land. Elle accueille un certain nombre de grands événements et de congrès (GamesCom, DMEXCO, Medienforum NRW) qui à leur tour contribuent au rayonnement du site et, par delà, du Land où coexistent aujourd'hui plusieurs pôles médiatiques présentant chacun un profil particulier. Ce polycentrisme comporte un grand potentiel de complémentarité à l'ère du numérique. C'est lui que la stratégie du Mediencluster NRW cherche à dynamiser en soutenant sa constitution en réseau -tout en en espérant des effets indirects sur le climat général d'innovation.
Optimiser le potentiel du polycentrisme sectoriel régional

\section{Rhénanie du Nord-Westphalie : spécialisations et pôles médias}

- TV : le Land est leader allemand dans cette activité, qui se concentre dans l'agglomération de Cologne. A la diffusion (WDR, DLF, DW, RTL, Super RTL, VOX, n-tv, Phoenix, GIGA, chaînes régionales...) et à la production (par exemple Endemol Allemagne) s'ajoute une dense offre de formations dans le domaine des médias, à la fois publique et privée, initiale et continue (universités, écoles supérieures spécialisées, Kunsthochschule für Medien, Hochschule Fresenius, Hochschule für Wirtschaft und Medien, Cologne Business School, Medienakademie Köln, RTL Journalistenschule für TV und Multimedia...).

- Médias en ligne : le Land est le fief des télécommunications, des TIC et de leurs applications, avec Deutsche Telekom (Bonn), Vodafone (Düsseldorf), mais aussi RTL interactive (Cologne), Onvista, Materna ou Firstgate Internet. A relever : les portails www.rtl.de (parmi les plus consultés avec 350 millions de pages imprimées par an). La plupart des portails sont cependant l'œuvre des éditeurs de presse (ainsi www.derwesten.de du groupe WAZ, Essen). Tous les ans est décerné à Cologne le prix Grimme Online Award.

- Livre : l'édition scientifique et spécialisée domine, et elle se concentre à Cologne où se trouve le siège de Wolters Kluwer Deutschland (il possède par exemple les éditions Luchterhand, Verlag für Deutsche Steuerberater, Verlag für ControllingWissen ou AnNoText), des éditions Verlag Dr. Otto Schmidt, du groupe Lübbe (spécialiste des bestsellers, éditeur de livres audio, un segment en plein boom outre-Rhin), Kiepenheuer und Witsch (littérature) ou encore de l'éditeur d'art Taschen.

- Presse quotidienne : l'offre est une des plus denses d'Allemagne avec 46 quotidiens (4,9 millions d'exemplaires) et 145 éditeurs de gratuits d'annonce (20,5 millions d'exemplaires). Les principaux acteurs sont : le groupe Westdeutsche Allgemeine Zeitung (WAZ, Essen), qui édite le quotidien régional du même nom et est leader allemand sur le segment des quotidiens vendus par abonnement, le groupe M. DuMont Schauberg (Cologne), avec Kölner Stadtanzeiger ou Berliner Zeitung, et le groupe von Holtzbrinck (Düsseldorf), avec le quotidien économique à diffusion nationale Handelsblatt et l'hebdomadaire Wirtschaftswoche.

- Presse périodique : elle se concentre à Cologne et offre une large gamme de contenus. Y sont édités par exemple : Echo der Frau, Frau aktuell (féminins, groupe WAZ), ou Emma (féministe historique, indépendant).

- Radio : Cologne est le siège du premier opérateur public Westdeutscher Rundfunk (WDR) qui produit 5 radios régionales, de la radio nationale allemande Deutschlandfunk (DLF) et de la radio-TV internationale Deutsche Welle (DW). La ville accueille tous les ans le salon Radio Day, co-organisé par les deux plus importantes centrales d'achat d'espace publicitaire radio d'Allemagne : RMS et ASS Radio. Oberhausen, dans la Ruhr, est le siège de l'opérateur radio NRW qui fédère les 45 radios locales privées du Land, et abrite un centre de formation initiale et continue dans les médias, détenu conjointement par le WDR, radio NRW et l'autorité de régulation de l'audiovisuel du Land (LfM) : la Deutsche Hörfunkakademie.

- Cinéma : production et distribution (à dominante PME) se concentrent dans l'agglomération de Cologne, avec les sociétés de production Pandora Film, Ariel Film, Colonia Film ou MMC Independant, ainsi que les distributeurs Media Luna Entertainment ou The Match Factory. L'agglomération est aussi le lieu de grandes manifestations: congrès Internationaler Filmkongress ou Film+, cérémonie de remise de prix KunstFilmBiennale Köln, et divers festivals (par ex. le Fantasy Filmfest).

- Jeux électroniques : deux pôles se dessinent autour des sociétés Electronic Arts, BMS Modern Games (Cologne) et Ubisoft (Düsseldorf) et d'un réseau de plus de 40 sociétés spécialisées dans le développement de programmes. S'y ajoutent de nombreuses manifestations : le World Cyber Games Grand Festival 2008 dont Cologne avait remporté l'organisation contre Pékin, Shanghaï et Stockholm, les eSport-Events annuels ou, depuis 2009, le salon européen GamesCom. Le développement a son propre award, réputé, décerné à Essen : le Deutscher Entwicklerpreis. Ce segment particulièrement dynamique s'implique aussi dans la formation : la Fachhochschule Köln offre ainsi depuis 2007 un master « Game development \& Research ».

- Industries musicales (supports) : Cologne mène, avec des sociétés comme EMI Music Germany, Groove Attack, Kompakt, Le Pop Music, Meyer Records ou une succursale de Sony BMG Ariola (Hambourg). Equipée de riches infrastructures (Kölnarena, Palladium, E-Werk, Live Music Hall, innombrables clubs), elle accueille concerts et festivals (par exemple le Ringfest, arrêté en 2006, ou le Summer Jam), ainsi que le congrès Conference c/o pop. S'y ajoute une large gamme d'agences de concerts (Peter Rieger, Concert tea, Dirk Becker Entertainment). Un segment est particulièrement dynamique : le jazz (et le swing, grâce notamment à la haute réputation de l'orchestre WDR Bigband et des formations décernées par la Kölner Musikhochschule).

- Affichage : Cologne est le siège de Ströer Out-Of-Home Media (numéro 1 allemand), Kölner Außenwerbung (société commune à celle-ci et à la régie municipale) et de la centrale Allemagne de J.C. Decaux.

- Publicité et marketing : Düsseldorf est le site leader en Allemagne, avec BBDO Group Germany, Grey Global Group Deutschland et la centrale Allemagne du groupe Publicis, la première familiale allemande du secteur (Eggert), ainsi que des succursales d'Ogilvy \& Mather, TBWA, Hakuhodo, McCann Erickson ou ddb. A Cologne gravitent autour de la TV en nombre les petites agences, dont Bruno Media, Ergo, Muehlhaus \& Moers, Weber Shandwick ou Oliver Schrott Kommunikation.

(Source des données : me:mi, Mediencluster NRW, 2007). 
Faire de la NRW le $n^{\circ} 1$

NRW : un retard qui vient du profil sectoriel du Land de l'innovation d'ici 2015

\section{Mediencluster : une innovation dans la politique d'innovation}

Car le gouvernement Rüttgers (coalition CDU/FDP) au pouvoir depuis 2005 s'est donné pour objectif de faire de la Rhénanie du Nord-Westphalie "le numéro 1 de l'innovation » en RFA à l'horizon 2015 - et donc aussi au sein des régions de I'UE. Si sa politique ne s'inscrit pas en rupture avec celles des gouvernements précédents (à majorité SPD), elle apporte néanmoins des inflexions nouvelles, (surtout au niveau des universités ou des technologies de pointe ; voir Bourgeois, 2007) d'autant que l'approche communautaire du champ cohésion et innovation a elle-même évolué entre-temps. La politique Rüttgers s'appuie sur quatre piliers : autonomie des établissements d'enseignement supérieur, promotion des technologies de pointe, stratégie de clusters et valorisation du site NRW selon une approche de marketing (RWI, 2007).

L'impératif prioritaire est de combler le retard relatif du Land en matière de R\&D. Ce retard est lié à la structure de ses activités issue de plusieurs décennies de mutations. Si les Länder du sud sont en tête, c'est que sur leur territoire se concentrent aujourd'hui les branches par définition les plus intensives en R\&D : l'automobile, l'électrotechnique et la construction mécanique. La région de la Ruhr, autrefois cœur industriel de l'Allemagne, s'est certes reconvertie, mais dans des secteurs moins intensifs en R\&D ou en technologies de pointe comme le commerce, l'énergie et les médias (RWI, 2008). Ainsi, tous secteurs confondus, $16 \%$ seulement des dépenses de R\&D de la RFA sont à mettre à l'actif du Land, qui accueille $16 \%$ seulement des ressources humaines afférentes (données 2006). Certes, la R\&D universitaire se distingue (le Land y consacre $0,43 \%$ de son PIB ; moyenne allemande : 0,42\%), mais celle des centres de recherche publics du Land $(0,27 \%$ du PIB) n'atteint pas encore, malgré de nets progrès, la moyenne allemande $(0,36 \%)$ ni le niveau des Länder du sud $(0,42 \%$ dans le Bade-Wurtemberg, leader incontesté). Mais cette forte part publique a pour corollaire l'insuffisance des dépenses privées en comparaison: seuls $14 \%$ du total allemand proviennent de NRW, alors que le Bade-Wurtemberg en génère le double. Enfin, on observe au sein du Land une forte disparité régionale entre d'un côté l'axe du Rhin (2,3 \% du PIB consacré à la R\&D) et d'autre part, la Westphalie (nord-est) et la région de la Ruhr (au centre, 1,3\% seulement).

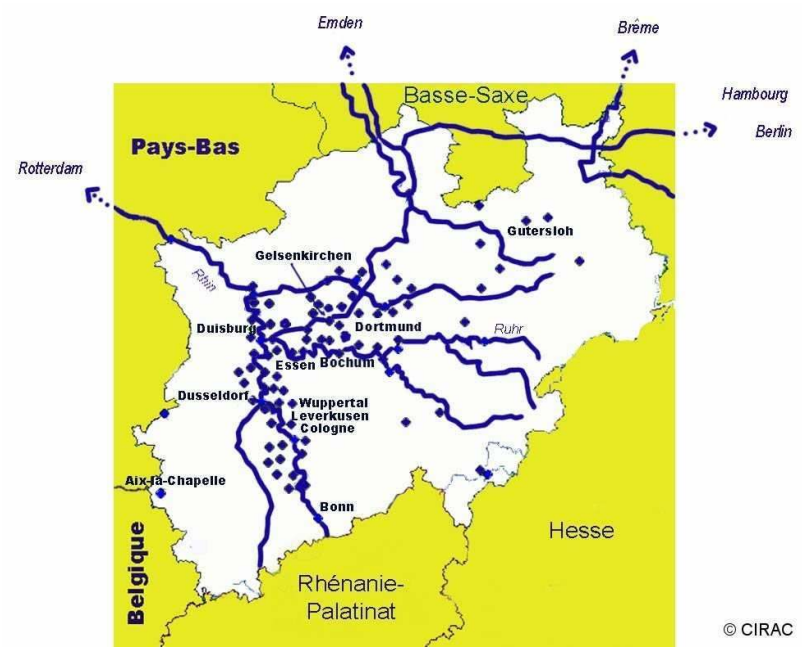

Un atout pour l'innovation la forte proportion d'étudiants
Dans un autre domaine clef en matière d'innovation aussi, la NRW marque le pas sur les deux Länder du sud: le système de formation. Ainsi, la proportion des diplômés du tertiaire dans la population est relativement faible en comparaison. Mais il s'agit là plus "de l'héritage de la politique éducative passée " que d'un déficit foncier car, souligne l'institut RWI dans son rapport 2008 d'évaluation du système d'innovation du Land; actuellement, le taux de nouvelles inscriptions dans le supérieur est supérieur à celui des Länder du sud; la NRW se classe 
juste après la Hesse. Et, avec 13,7 \%, la proportion des étudiants inscrits dans un établissement d'enseignement supérieur (universités, établissements d'enseignement théologique, artistique, pédagogique) dans la cohorte des 19-32 ans place la NRW en tête des Länder allemands (Bade-Wurtemberg: 10,5\%; Bavière : $9,9 \%$ ). Le Land mène également en ce qui concerne le taux de participation des 15-19 ans à la formation : il est de 91,9 \%. La NRW consacre actuellement un important effort à la modernisation de son système d'éducation et de formation (4,2 \% du PIB, soit plus que les deux Länder du sud, où cette part est inférieure à $4 \%$ ). Une seule ombre au tableau : la faible participation des salariés du Land à la formation continue. Au total, résume l'étude du DIW, si la NRW parvient à faire passer le taux d'inscription de ses bacheliers dans un établissement d'enseignement supérieur de $70 \%$ actuellement à $75 \%$, alors «le Land pourrait consolider et développer l'avantage compétitif dont il dispose déjà dans les grandes lignes », à savoir se positionner en site leader en matière de formation et d'innovation.

Le chemin est long et épineux, soulignait déjà l'institut RWI dans son rapport précédent (2007), rappelant qu'il " a fallu des décennies à la Bavière pour se transmuer d'économie agricole en site technologique ». Et il conseille vivement au gouvernement de ne pas céder aux attentes court-termistes d'une opinion guettant l'évolution annuelle des indicateurs établis d'innovation, mais de plutôt mener délibérément une stratégie au long cours, et globale. Celle-ci implique bien sûr le soutien massif des technologies de pointe ou porteuses d'avenir, mais sans négliger toutes ces technologies "conventionnelles », c'est-à-dire l'innovation incrémentale qui constitue par essence le cœur de la compétitivité du Mittelstand. Autrement dit : cette politique doit prendre en compte "les liens systémiques inhérents à l'innovation industrielle » que masquent généralement les nomenclatures statistiques car elles apportent une lecture fragmentée d'une réalité où les frontières entre ces différentes catégories sont par essence fluides.

Cette lecture, précise l'institut dans son rapport 2008, ne peut être que partielle, puisque les indicateurs usuels ne permettent pas de mesurer l'ampleur réelle du processus de transfert des technologies et savoirs - en particulier lorsqu'il s'agit de " ces savoirs tacites, c'est-à-dire non codifiés et non standardisés dont le transfert est impossible sans contacts interpersonnels directs ». De surcroît, tous les acteurs impliqués dans le processus d'innovation "se meuvent à l'intérieur d'un cadre réglementaire et institutionnel donné ». Dès lors, revêtent une importance centrale pour le processus d'innovation non seulement les performances individuelles de chacun des acteurs, mais également « leur interaction à l'intérieur de ce cadre comme leur imbrication respective dans des réseaux (de savoirs) régionaux, suprarégionaux ou internationaux ». L'institut RWI partage donc l'approche élargie de l'innovation qui tend à s'imposer en Allemagne. Elle est théorisée outre-Rhin par exemple par la Commission d'experts Recherche et Innovation (EFI), un think tank indépendant institué en 2006 par le gouvernement fédéral, qui la décrit comme un "ensemble global de processus dynamiques, souvent résumé sous le terme de 'destruction créatrice' " (rapport 2008, www.e-fi.de ; voir REA 90/09). Or dans cet ensemble de processus, le rôle clef revient à l'individu. La politique menée par le gouvernement du Land au titre du programme EFRE objectif 2 tente de concilier cette multitude d'approches.

Le point crucial reste que «le développement d'une dynamique d'innovation dépend de manière décisive de la compétence sectorielle et de la créativité des individus impliqués dans la $R \& D$ »(RWI, 2007). Concrètement, cela signifie que, la capacité d'innovation d'une région ou d'un Land reposant non seulement sur l'investissement public ou celui des entreprises, mais bien plus encore sur la présence d'un capital humain adéquat, celui-ci doit être placé au centre des préoccupations. II doit l'être de plusieurs manières. En disposant d'un système de formation de qualité, bien sûr. Mais les ressources humaines hautement qualifiées sont par définition enclines à migrer vers les sites les plus attractifs et, au sein de l'Allemagne, cette mobilité profite surtout aux Länder du sud (voir REA 88/08). Pour "développer efficacement » la compétitivité de la NRW dans les technolo-
La politique doit intégrer l'innovation incrémentale...

... et se souvenir que l'innovation est un processus parent de la 'destruction créatrice'

Entretenir un environnement attractif pour la créativité 
gies d'avenir, " il importe donc de maintenir dans le Land et d'y attirer » les chercheurs de pointe, insiste le RWI (2007). La politique d'innovation du Land doit donc aussi consister à entretenir un environnement attractif et à le faire savoir.

Le programme Ziel2.NRW (2005-2013), doté de 2,5 milliards $€$ au total *)

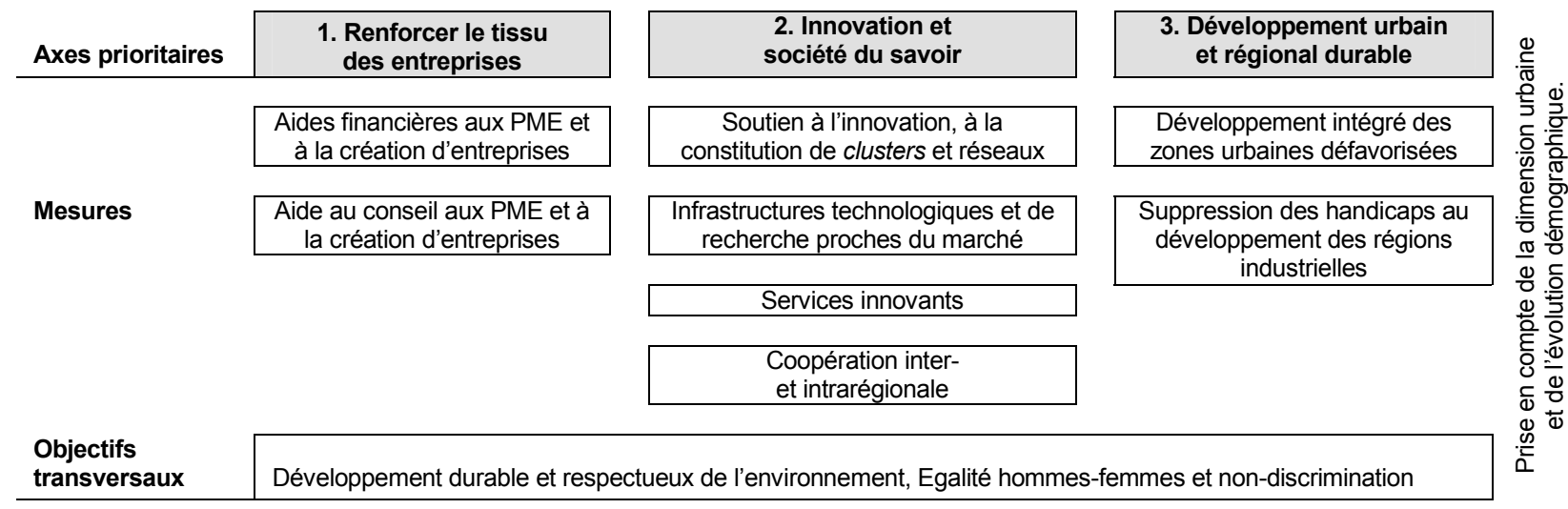

Source : www.ziel2-nrw.de/1_Ziel2-Programm/1_Programmziele/index.php. *) frépartis ainsi : $1: 20$ \%, 2 : 50 \%, 3 : 30 \%.

Une structure polycentrique des activités innovantes

Pour ce faire, le gouvernement mise sur les atouts du Land. En premier lieu sur les atouts structurels. Alors que le potentiel global d'innovation de la Bavière concurrente se concentre presque entièrement sur l'agglomération de la capitale (Munich), en Rhénanie du Nord-Westphalie, il se répartit sur l'ensemble du territoire : Aix-la-Chapelle à l'ouest, Paderborn, Münster ou Bielefeld au nord-est, région de la Ruhr au centre. En NRW, les « activités liées à l'innovation présentent une structure polycentrique », souligne l'institut économique RWI d'Essen (RWI, 2007). C'est là que l'approche en termes de réseaux prend tout son sens puisqu'il s'agit, dans la définition des politiques dédiées à l'innovation comme à la cohésion territoriale, de prendre en considération cette diversité comme l'un des facteurs clefs de la dynamique économique. A la différence de la plupart des autres Länder, la NRW dispose d'un atout décisif à l'appui de l'approche de mise en réseau des capacités comme du potentiel : un réseau d'infrastructures de transport particulièrement dense et performant assurant la mobilité des acteurs.

Industries culturelles et créatives en Rhénanie du Nord-Westphalie (2007)

\begin{tabular}{|c|c|c|c|c|c|c|}
\hline & Entreprises $^{\text {a) }}$ & $(\%$ de $A+B)$ & CA (mio $€)$ & $(\%$ de $A+B)$ & Emplois $^{\text {b) }}$ & $(\%$ de $A+B)$ \\
\hline A. Industries culturelles (total) & 37711 & 75 & 24802 & 70 & 133802 & 63 \\
\hline Livre/édition musicale & 2056 & 4 & 10592 & 30 & 28942 & 14 \\
\hline Production audiovisuelle et cinéma & 1843 & 4 & 1098 & 3 & 9937 & 5 \\
\hline Opérateurs radio et TV & 226 & 0 & 3048 & 9 & 13817 & 7 \\
\hline Arts/spectacles vivants & 8807 & 18 & 1500 & 4 & 16736 & 8 \\
\hline Agences et bureaux d'information & 4355 & 9 & 328 & 1 & 5656 & 3 \\
\hline Musées, expositions & 298 & 1 & 127 & 0 & 775 & 0 \\
\hline Commerce de produits culturels & 1884 & 4 & 1370 & 4 & 8377 & 4 \\
\hline Bureaux d'architectes & 8361 & 17 & 1517 & 4 & 20970 & 10 \\
\hline Bureaux de design & 9882 & 20 & 5222 & 15 & 28591 & 13 \\
\hline$\%$ dans le total des activités du Land & $5,6 \%$ & & $1,9 \%$ & & $2,1 \%$ & \\
\hline B. Industries créatives (total) & 12590 & 25 & 10538 & 30 & 78095 & 37 \\
\hline Publicité & 4451 & 9 & 5258 & 15 & 12716 & 6 \\
\hline Logiciels et jeux & 8139 & 16 & 5279 & 15 & 65379 & 31 \\
\hline Industries cult. et créatives ( $\Sigma \mathrm{A}+\mathrm{B})$ & 50301 & 100 & 35340 & 100 & 211897 & 100 \\
\hline$\%$ dans le total des activités du Land & $7,5 \%$ & - & $2,6 \%$ & - & $3,3 \%$ & - \\
\hline [PM : total activités éco. du Land] & 668059 & - & 1334895 & - & 6332030 & - \\
\hline
\end{tabular}

Source des données: NRW.Bank. a) entreprises soumises à impôt, au CA > $17500 €$; b) indépendants et salariés soumis à cotisations sociales (plus de 15 heures hebdomadaires, revenus mensuels $>400 €$ ), sans les salariés des théâtres, musées et bibliothèques du secteur public.

Concentration des industries culturelles dans la métropole Ruhr/Rhin
Elle en présente d'autres, moins communément pris en considération dans le cadre des politiques d'innovation : d'abord une forte concentration urbaine. La région métropole Ruhr/Rhin se distingue par sa densité de population (2 200 habi- 
tants $/ \mathrm{km}^{2}$ en moyenne dans le bassin de la Ruhr), et on y compte 6 villes de plus d'un demi-million d'habitants, dont Cologne, Duisburg, Düsseldorf et Essen. Ensuite, cette région métropole à forte concentration d'activités est aussi le principal pôle d'industries culturelles, avec près des trois quarts des entreprises du Land (80 \% du CA de la 'branche'). A l'échelle de la RFA, la NRW abrite le quart des entreprises du secteur et génère le quart de son $C A$, estimé à 61 milliards $€$ (2,6 \% du PIB allemand) par le ministère fédéral de l'Economie qui place cette 'branche' en $3^{\mathrm{e}}$ position après la construction mécanique et l'automobile, pesant respectivement 74 et 71 milliards $€$ en 2006 (BMWI, 2009).

Cette concentration joue un rôle clef dans l'attractivité globale du site (elle fidélise le capital humain et attire les investisseurs) et apporte une contribution non moins importante à la dynamique économique régionale. Cette contribution est à la fois directe (création d'entreprises, d'emplois, revenus fiscaux, développement du secteur touristique...) et indirecte : elle est valorisante en termes de positionnement international et d'image - élément primordial pour la Ruhr qui n'a pas entièrement réussi encore à se défaire de sa réputation pourtant obsolète de "pays noir ». C'est donc là un atout à la fois économique et en termes de notoriété qu'il s'agit de développer. Et le Land s'y emploie, mettant à profit notamment le fait qu'Essen soit capitale culturelle de l'Europe en 2010 et qu'un de ses anciens puits de mine (Zeche Zollverein) soit classé au patrimoine de l'Unesco.

Industries culturelles et créatives : un précieux « marché d'idées » pour l'économie

Certes, cette 'branche' des industries culturelles et créatives reste très floue, étant donné que les diverses nomenclatures statistiques, nationales ou communautaire, par définition statiques puisqu'elles recensent l'existant sur la base de catégories historiques, ne sont guère adaptées à une situation évolutive comme celle de ces activités liées de près ou de loin à la création intellectuelle et au marché à la fois. C'est une des raisons pour lesquelles ces activités ne sont pas ou peu prises en considération jusqu'ici parmi les grands indicateurs économiques. Rappelons qu'il a fallu des décennies pour que commencent à s'esquisser les contours statistiques d'un secteur des services alors que, dans le fil des grandes mutations de l'économie, les activités s'étaient largement tertiarisées.

Aujourd'hui, on commence certes à entrevoir l'impact de ces industries culturelles et créatives sur la dynamique économique, mais dans cette phase préalable, un certain nombre de nomenclatures coexistent (voir par exemple les rapports du Bundestag, 2007, ou celui du BMWi, 2009), et il faut donc manier les chiffres avancés par les différentes sources avec prudence. La Nomenclature statistique des activités économiques dans la Communauté européenne (NACE) permet certes les comparaisons, mais étant alimentée par les statistiques des Etats membres, offre une vision réductrice du champ: la catégorie NACE Edition rassemble musique et livre, deux secteurs distincts dans la nomenclature allemande; quant à la catégorie "creative industries " développée en Grande-Bretagne et à la base des travaux communautaires sur la thématique, elle ne sépare pas, contrairement aux données allemandes, activités commerciales, privées, et dépenses publiques. En Allemagne même, plusieurs définitions ont cours (dont celles du Bundestag, de la Conférence des ministres de l'Economie ou de la Conférence permanente des ministres de la Culture).

Enfin, plus fondamentalement, se pose la question de l'approche de ces activités. La catégorie industries musicales, par exemple, ne prend en compte le plus souvent que les activités liées aux supports ; comment et où classer la 'production' des contenus, sans lesquels il n'y aurait ni pressage de CD ou DVD, ni diffusion d'émissions ni organisation de concerts ni marché de discothèques ? Comment définir les métiers? Quel traitement réserver à la formation des créateurs? etc.

Par delà la question du périmètre statistique, ce qui nourrit actuellement l'intérêt des différentes parties prenantes pour les industries culturelles et créatives, c'est la profonde conviction qu'elles " sont en train de développer une complémentarité nécessaire dans une infrastructure économique moderne » et qu'elles constituent un "'marché et un laboratoire d'idées' susceptible d'ouvrir de précieuses perspectives pour l'évolution globale de l'économie » (CBC, KWF, Prognos, 2009).

En Allemagne, la Rhénanie du Nord-Westphalie fait là figure de précurseur : elle a découvert le potentiel économique des industries culturelles - dont des médias - dès 1990, les considérant comme "une branche dont la croissance était susceptible de soutenir la reconversion de l'économie industrielle (charbon et acier) vers l'économie du savoir et la société de l'information ", souligne le Bundestag dans son rapport sur la culture en Allemagne (2007). D'autres Länder ou agglomérations ont suivi son exemple; mais alors que leur objectif était principalement de développer le tourisme (Schleswig-Holstein) ou de se positionner en challenger culturel de Paris (Berlin), la Rhénanie du Nord-Westphalie cherchait avant tout à «légitimer une nouvelle politique structurelle », explique le même rapport.
Un gisement de créativité à exploiter mieux encore 
Développer la convergence à l'ère du numérique

Mediencluster NRW : réaliser la convergence territoriale aussi

Intégrer les entreprises dans des réseaux d'échanges...

... et former la relève créative à la convergence
La même région métropole présente par ailleurs une constellation unique, au potentiel de compétitivité indéniable dans l'économie du savoir. Autour de l'opérateur historique des télécommunications et de ses nouveaux concurrents, nés avec l'ouverture de ce marché à la concurrence au milieu des années 1990, s'était constitué par grappage un pôle dynamique s'étendant de la téléphonie fixe et mobile à l'ensemble des TIC (des composants aux services), tiré par l'essor des applications informatiques au fur et à mesure que montaient en puissance les services aux entreprises (du B2B à la logistique en passant par les centres d'appel). Il comprend aujourd'hui toute une série d'activités à l'interface entre hardware et software, entre TIC et médias (comme les jeux électroniques, un segment en forte croissance). Ces activités de moyennes technologies dans l'ensemble, de nature incrémentale, sont néanmoins intensives en R\&D et, plus important encore, s'accompagnent d'une forte créativité. Si un cluster TIC a été lancé dans le cadre de la politique d'innovation, et si un axe de la politique économique générale du Land vise à dynamiser le secteur des industries culturelles et créatives (notamment via l'appel à projets Create.NRW 2009 lancé en mars dernier), un autre consiste à se baser sur la complémentarité ou la convergence observée entre les TIC au sens large et les médias (contenus) pour mieux en faire émerger les synergies et en optimiser le potentiel économique et créatif. Dans une Allemagne où les médias sont non seulement une économie prospère mais jouissent aussi d'une bonne image comme de la confiance des consommateurs, le secteur se prête bien de surcroît pour une campagne d'image.

$\mathrm{Si}$, d'une manière générale, le concept de cluster est vendeur (c'est le maître mot des politiques d'innovation, communautaire et nationales), il n'en est pas moins chargé de sens en l'occurrence. Car à l'échelle de la région métropole Ruhr/Rhin s'était institué au fil de l'histoire (du fait aussi que la législation rattache les télécommunications à l'économie, donc au Bund, les médias à la culture, donc aux Länder) une sorte de partage du travail entre les indépendants et sociétés produisant des contenus (Cologne, Düsseldorf) et ceux produisant supports ou services au client (Ruhr). Ce découpage était favorisé en outre par le fait que les médias (contenus) relèvent du droit constitutionnel et que, contrairement aux télécommunications, ils échappent largement au droit commun de la concurrence (voir REA 75/06). Réunir ces deux 'mondes parallèles' au sein d'un cluster revient donc à mieux prendre en compte les réalités de l'évolution des marchés respectifs dans l'attente d'une vitalisation à la fois du tissu économique et de la créativité en soi.

Enfin, les médias comme l'ensemble des industries culturelles et créatives se distinguent des autres 'branches' en ce sens que, si on y relève quelques géants (grands opérateurs de TV ou de télécommunications, groupes multimédias), l'essentiel des activités est le fait de très petites entreprises et de nombreux indépendants. La structure du tissu des entreprises des industries culturelles et créatives en NRW est parlante : 94,5\% d'entre elles réalisent un CA inférieur à 1 million $€$; dans ce groupe, 63,3\% un CA inférieur à $100000 €$ (données 2006, NRW.Bank). Mais ces PME ne réalisent que $16,9 \%$ du CA de la branche, les 1,2\% de géants (CA supérieur à 5 millions $€$ ) se taillant la part du lion avec $70,6 \%$ du total. Or, comme les petites structures constituent l'essentiel du vivier créatif ou innovant, une stratégie de soutien ciblée s'impose, et la banque publique régionale NRW. Bank comme le gouvernement leur ont dédié, dans le cadre de leur politique générale de promotion des PME, des programmes de soutien spécifiques (aides financières, conseils, accompagnement). Mais ce n'est pas le seul aspect considéré. Pour assurer la pérennité de leur créativité, ces petites structures souvent isolées ont besoin de mieux se rapprocher des grands groupes qui disposent de la puissance financière mais sont en quête continuelle d'innovation, et aussi de s'intégrer dans des réseaux (partenariats commerciaux ou simplement échanges d'idées), a fortiori dans un Land où cette branche est par définition polycentrique.

Dans le même objectif, il faut que les créateurs/innovateurs des médias aient accès dans une plus large mesure à une offre de formation continue adaptée à la convergence - une priorité "dans un Land où la convergence n'est pas théo- 
rique, mais relève de la pratique quotidienne ", comme le souligne Andreas Krautscheid, ministre des médias du Land et également ministre en charge des relations avec le Bund et des Affaires européennes (epd medien, 07-06-2008). Dans la formation initiale, audiovisuel et Internet restent encore trop des univers parallèles. Mais comme la définition de nouveaux profils professionnels est un processus très long outre-Rhin, puisqu'il implique une multitude d'institutions, le gouvernement du Land met à profit le Mediencluster NRW pour amener les acteurs de la formation à moderniser leur offre. "Dès années s'écouleraient avant que nous réussissions à définir de nouveaux profils avec la chambre de commerce. Nous préférons donc ajouter aux formations initiales de qualité [offertes dans le Land; IB], des modules [de formation continue] axés sur la convergence ", affirme le ministre. Et il résume ainsi les deux approches du cluster médias que sont "la mise en réseau et l'innovation » : "la première sera plutôt centrés sur les technologies, la seconde sur la formation convergente ».

Le Mediencluster NRW s'inscrit dans la même démarche que les 15 autres clusters lancés dans le Land (automotive, chimie, énergie, logistique ou biotechnologies, mais aussi industries culturelles) : " elle promeut la coopération entre entreprises, centres de recherche et pouvoirs publics tout au long des chaînes de création de valeur " de chacun des secteurs retenus (www.exzellenz.nrw.de). Les compétences du gouvernement en la matière sont réparties entre les divers ministères: celui de l'Economie, des PME et de l'Energie est par exemple en charge de la logistique, mais aussi des industries culturelles; celui de l'Innovation, de la Recherche et des Technologies, en charge des technologies médicales, des nouveaux matériaux ou des biotechnologies. Quant aux clusters médias et TIC, ils relèvent tous deux de la chancellerie, donc du ministre-président. Mais si le gouvernement a décidé d'initier un cluster médias, il s'est contenté d'identifier une priorité sectorielle, refusant d'entrer plus avant dans la définition des projets et thèmes à soutenir. "II n'est pas possible de les inventer à l'échelon de la chancellerie, ils ne peuvent qu'émaner de la branche elle-même " (Krautscheid, 2008). Et, bien qu'il en assure une partie du financement, il n'en assure donc pas la tutelle non plus. En effet, explique l'institut RWI (2007) : " en règle générale, le soutien de l'Etat a un effet de catalyseur sur les évolutions, mais celles-ci sont pilotées en dernier ressort par les processus inhérents au marché ».

Le top down (identification de la branche et lancement de la mesure) cède donc une large place au bottom up au niveau de la gestion des projets comme du management de la tête de réseau. Et plus généralement, par l'implication autonome de la branche tout au long de la genèse du cluster entre l'annonce officielle de cette mesure dans le discours d'ouverture du Medienforum NRW 2007 prononcé en juin par le ministre-président du Land, Jürgen Rüttgers, la remise d'un rapport sur le potentiel d'avenir des différents segments des médias par l'institut me:mi en décembre de la même année, puis l'institution d'une commission des médias (Medienbeirat) composée de professionnels et dont la mission est d'être un forum d'idées comme de prospecter les évolutions de la branche. C'est sur la base des travaux de l'institut me:mi (voir ci-après l'entretien avec son directeur, le Prof. Werner Schwaderlapp) qu'a été lancé le 14 août 2008 le premier appel à projets.

Quelque 170 entreprises, universités et centres de recherche du Land y avaient répondu, présentant au total 72 projets en coopération. Ce retour a permis d'identifier " six thèmes stratégiques " pour le site NRW, comme le formule le rapport d'évaluation réalisé par le cabinet Deloitte pour le compte du gouvernement du Land: jeux électroniques (branche dynamique, fortement présente en NRW), Internet mobile (fort potentiel commercial), services d'information locale (forte demande), TV mobile (diversification en cours de la TV classique), réseaux sociaux (95\% des moins de 20 ans y participent ; enjeux stratégiques pour le marketing) et offre de formation (manque de personnel qualifié dans le secteur des TIC, notamment dans le segment des jeux électroniques). Le 8 février 2009 a été publiée la liste de 12 projets sélectionnés par un jury indépendant créé ad hoc et composé lui aussi de représentants de la branche en février 2009.
Le Mediencluster NRW est placé sous la responsabilité du ministre-président...

... mais son pilotage et le choix des projets relèvent des acteurs de la branche

L'appel à projets a permis d'identifier 6 thèmes stratégiques 
Les 12 projets retenus en 2009 à l'issue du premier appel à projets (soutien du Land : jusqu'à 8 millions $€$ )

\begin{tabular}{|c|c|}
\hline Responsable/Coordination & Intitulé/Description \\
\hline $\begin{array}{l}\text { AIM KoordinationsCentrum für Ausbildung in Medienberufen in Trägerschaft der sk } \\
\text { stiftung jugend und medien (Cologne). Partenaires : direction médias et } \mathrm{CCl} \text { de Cologne. }\end{array}$ & $\begin{array}{l}\text { Dispense de qualifications (formation initiale et } \\
\text { continue) dans le secteur des jeux électroniques. }\end{array}$ \\
\hline Cinematic Games GmbH (Bochum). Partenaire : Nevigo GmbH (Bochum) & $\begin{array}{l}\text { Développement de la technologie « Articy » et du } \\
\text { jeu vidéo « Sapiens ». }\end{array}$ \\
\hline $\begin{array}{l}\text { DiscVision GmbH (Paderborn). Partenaires : Nacamar GmbH (Düsseldorf) et Fraunhofer } \\
\text { Institut IAIS (St. Augustin). }\end{array}$ & TAT - Targetted Advertisement . \\
\hline $\begin{array}{l}\text { GCC - Games Campus Cologne GmbH (Cologne). Partenaires : Catni Studios GmbH et } \\
\text { Deutsche Sporthochschule (Cologne). }\end{array}$ & $\begin{array}{l}\text { Wie fit bist du ? (développement de logiciels de } \\
\text { prévention santé pour enfants et adolescents) }\end{array}$ \\
\hline $\begin{array}{l}\text { GRUNDY Light Entertainment GmbH (Cologne). Partenaires : Entertainment Media } \\
\text { Verlag et ville de Cologne. }\end{array}$ & Création d'un European Games Award. \\
\hline $\begin{array}{l}\text { Projektgruppe Cologne Game Lab an der Fachhochschule Köln (Cologne). Partenaires : } \\
\text { Fachhochschule et ifs internationale filmschule (Cologne). }\end{array}$ & $\begin{array}{l}\text { Cologne Game Lab (formation initiale et continue, } \\
\text { recherche). }\end{array}$ \\
\hline $\begin{array}{l}\text { LIVN GmbH (Wuppertal). Partenaires : Buchdruckerei und Verlag F. Winter GmbH \& Co. } \\
\text { KG (Steinfurt), Ibbenbürener Volkszeitung et Ibbenbürener Vereinsdruckerei GmbH (Ib- } \\
\text { benbüren), Verlag J. Mergelsberg GmbH \& Co. KG (Borken), innowise GmbH (Duis- } \\
\text { burg), livn GmbH (Wuppertal), Varion GmbH (Krefeld). }\end{array}$ & $\begin{array}{l}\text { Vor-Ort-TV in NRW (développement d'une plate- } \\
\text { forme intégrée associant applications IPTV indivi- } \\
\text { duelles, régionales avec des services en ligne } \\
\text { fixes et mobiles). }\end{array}$ \\
\hline $\begin{array}{l}\text { Pixelpark AG (Cologne). Partenaires : Spin-TV/Firts-Entertainment, Sevenval, Commu- } \\
\text { nology, Dialogs Software, RIAS-Rhein-Ruhr Institut für angewandte Systeminnovation. }\end{array}$ & $\begin{array}{l}\text { LocalMobile.NRW (développement d'une applica- } \\
\text { tion mobile locale et intelligente offrant informa- } \\
\text { tions contextuelles et contenus mobiles } 2.0 \text { ). }\end{array}$ \\
\hline $\begin{array}{l}\text { Verband Druck + Medien NRW e.V. (Düsseldorf). Partenaires : Arbeitgeberverband } \\
\text { Druck+Medien NRW e.V. et Bergische Universität Wuppertal. }\end{array}$ & $\begin{array}{l}\text { Print goes media (Offensive de formation et d'in- } \\
\text { novation à la convergence pour le Land). }\end{array}$ \\
\hline $\begin{array}{l}\text { ZMGK - Zentrum für Medien und Gesundheitskommunikation e.V. (Cologne). Parte-" } \\
\text { naires : Université de Cologne (département psychologie des médias et psychologie } \\
\text { sociale), gewi-institut, Europa-Fachhochschule Fresenius (Cologne). }\end{array}$ & $\begin{array}{l}\text { Identification de nouveaux marchés à l'interface } \\
\text { entre les secteurs des médias et de la santé. }\end{array}$ \\
\hline JEP Productions Jürgen Engeloff e.K. (Cologne). Partenaire : Glamus GmbH. & $\begin{array}{l}\text { CrimeNein (plateforme interactive d'information } \\
\text { pour enfants et adolescents sur le thème crimina- } \\
\text { lité/prévention/sécurité). }\end{array}$ \\
\hline $\begin{array}{l}\text { Ifs internationale filmschule köln (Cologne). Partenaire : TAG/TRAUM Filmproduktion } \\
\text { GmbH \& Co. KG (Cologne). }\end{array}$ & $\begin{array}{l}\text { Sportlich spielend lernen - ssl (développement de } \\
\text { prototypes de jeux éducatifs et de formations ini- } \\
\text { tiales et continues dans le domaine de jeux au } \\
\text { contenu narratif non linéaire, proche du réel, et } \\
\text { mettant en œuvre des composants Exer-Games). }\end{array}$ \\
\hline
\end{tabular}

Source : Staatskanzlei NRW (www.nrw.de), Deloitte, 2009.

Management professionnel pour la tête de réseau
Ce n'est qu'ensuite qu'a été créée la société chargée du pilotage du cluster : la Mediencluster NRW GmbH. Bien qu'il s'agisse d'une filiale de la NRW.Bank qui gère les fonds alloués, cette société est indépendante. Elle est opérationnelle depuis l'entrée en fonctions de son directeur le 20 juillet 2009, Marc Ziegler, professionnel du conseil aux entreprises dans les domaines médias, Internet et télécommunications. Cette structure de pilotage, dotée d'une mission d'accueil et d'information, œuvrera également à la promotion du site médias, en particulier à l'échelle internationale.

LA PROMOTION DES MÉDIAS qui fut, voici vingt ans, plus un outil de communication qu'une mesure consistante de la politique de reconversion, se transmue aujourd'hui en un levier d'innovation parmi d'autres pour le Land. "La politique économique du gouvernement se réfère au modèle d'une économie basée sur la créativité. Sa mission est dès lors de promouvoir de manière ciblée, par le soutien à l'innovation et à la constitution de réseaux les marchés porteurs comme celui des médias ». C'est ainsi que J. Rüttgers décrivait la nouvelle approche de son gouvernement lors de l'ouverture du congrès Medienforum 2007, reconnaissant l'importance des médias pour les gisements d'innovation du Land. Si, comme l'affirme l'institut RWI (2007), les clusters sont "inadaptés pour servir de 'remède miracle' » dans le cadre d'une politique d'innovation, ils peuvent être justifiés lorsqu'ils visent à développer des pôles préexistants et qu'ils sont "faits sur mesure ». Or le Mediencluster NRW répond d'autant plus pleinement à cette exigence de consolidation des atouts, tout en misant sur l'effet levier de ce secteur créatif sur la compétitivité globale du site, que le gouvernement a 
su se saisir d'une opportunité idéale : la NRW (avec la Sarre) a décidé la fin définitive de l'extraction de la houille (et donc des subventions) d'ici 2018.

Le Land a donc aussi les moyens pour innover en matière de politique d'innovation. " Pour l'agglomération la plus peuplée d'Allemagne - la région de la Ruhr l'adieu au charbon, incontournable bien qu'indéniablement douloureux, devrait être le symbole de son entrée massive dans la société du savoir du XXI siècle », recommande l'institut RWI (2007). II y voit une " chance » que doit saisir la politique d'innovation de la NRW pour " enfin réaliser dans cette région ses objectifs fonciers : permettre le développement du capital humain sur une large base en même temps que l'épanouissement des talents individuels, l'émergence de l'excellence dans la recherche et le soutien aux technologies nouvelles comme aux technologies 'anciennes', mais vitales ».

\section{Indications bibliographiques}

- BouRgeoIS I., «Rhénanie du Nord-Westphalie : reconversion du charbon au high-tech », in BouRGEOIS I., (dir.) Allemagne : compétitivité et dynamiques territoriales, CIRAC, Cergy-Pontoise, 2007

- BMWı, Gesamtwirtschaftliche Perspektiven der Kultur- und Kreativwirtschaft in Deutschland, Forschungsbericht $n^{\circ}$ 577, février 2009 (www.bmwi.de)

- CAHN J.-G., « Bientôt sauvés par notre créativité? », Friedlandpapers n 13, janvier 2009

- Deloitte, Minister für bundesangelegenheiten, Europa und Medien des Landes NRW, Innovative Ideen. Medienstandort Nordrhein-Westfalen - Förderwettbewerb Medien.NRW, juin 2009 (www.deloitte.com/de)

- Die LANDESREgIERUNG NRW, EU-Strukturfonds für NRW und das NRW-Programm Ländlicher Raum. Eu-Förderung 2007-2013 (www.nrw.de)

- Im Blickpunkt : Medienland NRW 2009, juin 2009 (www.media-nrw.de)

- Medien.NRW. Gesucht: Die besten Ideen für Innovation, Konvergenz und Vernetzung im Medienbereich. Wettbewerbsaufruf und Leitfaden zum Förderwettbewerb, appel à projets publié conjointement par la Staatskanzlei du Land, le Ministerium für Bundesangelegenheiten, Europa und Medien et le Ministerium für Wirtschaft, Mittelstand und Energie, Düsseldorf, 14 août 2008 (www.ziel2nrw.de et www.medien-nrw.de)

- Minister für bundesangelegenheiten, Europa und Medien des Landes NRW, Medienausbildung in NRW. Ausbildungsberufe und Studiengänge, mai 2009 (www.mbem.nrw.de)

- Ministerium für WiRTSchaft, MitTelstand Und Energie des Landes NRW, Regionale Wettbewerbsfähigkeit und Beschäftigung 2007-2013 (EFRE - Ziel 2). Operationelles Programm (EFRE) für das Ziel „Regionale Wettbewerbsfähigkeit und Beschäftigung“ für Nordrhein-Westfalen nach Artikel 37 der Verordnung (EG) Nr. 1083/2006 des Rates vom 11. Juli 2006, juin 2007

- NRW.BANK (ed.), Kultur- und Kreativwirtschaft. Ökonomische Impulse für Nordrhein-Westfalen, (www.nrwbank.de)

- CBC, KWF, Prognos, Kultur- und Kreativwirtschaft: Ermittlung der gemeinsamen charakteristischen Definitionselemente der heterogenen Teilbereiche der ,Kulturwirtschaft' zur Bestimmung ihrer Perspektiven aus volkswirtschaftlicher Sicht. Endbericht, Cologne, Brême, Berlin, février 2007

- SchWADERLAPP W. et al., Mediencluster NRW: Innovationen fördern, Strukturen bilden. Ausgangslage und Schwerpunkte für die Wirtschaftsförderung am Standort. Studie des memi-Instituts gefördert durch die Staatskanzlei NRW, Cologne, décembre 2007 (www.memi-koeln.de)

- Deutscher Bundestag, Schlussbericht der Enquête-Kommission „Kultur in Deutschland“, BTDrucksache 16/7000, décembre 2007

- KEA EuRopean AffaIRS, The Impact of Culture on Creativity. A Study prepared for the European Commission (Directorate-General for Education and Culture), juin 2009. 